\title{
ChemComm
}

Check for updates

Cite this: Chem. Commun., 2020, 56,6098

Received 11th February 2020 Accepted 23rd April 2020

DOI: $10.1039 / \mathrm{d} 0 \mathrm{cc} 01085 \mathrm{e}$

rsc.li/chemcomm

\section{A versatile cholera toxin conjugate for neuronal targeting and tracing $\dagger$}

\author{
Jessica L. Haigh, (D ab Daniel J. Williamson, ${ }^{a}$ Emma Poole, (iD a Yuan Guo, (D) c \\ Dejian Zhou, (iD a Michael E. Webb, (D) a Susan A. Deuchars, (ID b Jim Deuchars (D)*b \\ and W. Bruce Turnbull (iD) *a
}

Tracing of neurons plays an essential role in elucidating neural networks in the brain and spinal cord. Cholera toxin B subunit (CTB) is already widely used as a tracer although its use is limited by the need for immunohistochemical detection. A new construct incorporating non-canonical azido amino acids (azido-CTB) offers a novel way to expand the range and flexibility of this neuronal tracer. Azido-СТВ can be detected rapidly in vivo following intramuscular tongue injection by 'click' chemistry, eliminating the need for antibodies. Cadmium selenide/zinc sulfide (CdSe/ZnS) core/shell nanoparticles were attached to azido-CTB by strain-promoted alkyne-azide cycloaddition to make a nano-conjugate. Following tongue injections the complex was detected in vivo in the brainstem by light microscopy and electron microscopy via silver enhancement. This method does not require membrane permeabilization and so ultrastructure is maintained. Azido-CTB offers new possibilities to enhance the utility of CTB as a neuronal tracer and delivery vehicle by modification using 'click' chemistry.

Cholera toxin produced by Vibrio cholerae is usually an infectious agent of the gut, but the toxin also has the potential to be developed for more versatile tracing and targeting of neurons. It is formed of two main parts: the catalytically active A subunit and the non-toxic pentamer of B subunits (CTB) that facilitate cell entry via binding ganglioside GM1.1,2 GM1 is ubiquitously expressed in vertebrates but is found in high relative abundance in the nervous system, including in the periphery of motor neurons at the neuro-muscular junction; for this reason CTB has been used extensively as a neuronal tracer. ${ }^{3-6}$ It is usually detected via antibody-mediated detection using fluorescence or 3'-Diaminobenzidine (DAB) immunohistochemistry, ${ }^{7-9}$ but direct fluorophore conjugates are also available $\mathrm{a}^{10,11}$ as well as CTB-horseradish

\footnotetext{
${ }^{a}$ School of Chemistry and Astbury Centre for Structural Molecular Biology, University of Leeds, Leeds LS2 9JT, UK. E-mail: w.b.turnbull@leeds.ac.uk

${ }^{b}$ School of Biomedical Sciences, Faculty of Biological Sciences, University of Leeds, Leeds LS2 9JT, UK

${ }^{c}$ School of Food Science and Nutrition, University of Leeds, Leeds LS2 9JT, UK

$\dagger$ Electronic supplementary information (ESI) available. See DOI: 10.1039/ docc01085e
}

peroxidase (HRP) conjugates for detection via peroxidase chemistry. ${ }^{4,12}$ The ability to detect the tracer directly without the need of antibodies is advantageous. It does not require an amplification step and so the labelling process is quicker, and cheaper as no antibodies are required. Detecting proteins without immunohistochemistry also provides more scope for labelling multiple proteins without the need to consider crossreactivity of primary and secondary antibodies as well as removing issues with antibody batch variability and limited shelf-life. Existing fluorescein-CTB conjugates are prone to bleaching, CTB-HRP conjugates are not compatible with immunofluorescence methods and neither enables easy detection by electron microscopy as membranes must be permeabilised to enable detection.

One method of detecting proteins without using antibodies is to use a bioorthogonal reaction such as copper-catalysed azide-alkyne cycloaddition (CuAAC) commonly referred to as 'click chemistry'. ${ }^{13-15}$ As the azide group is small it can be incorporated into probe molecules without interfering with their behaviour in live organisms, while providing the opportunity for subsequent conjugation to fluorescent probes. Alternatively, strain-promoted azide-alkyne cycloaddition (SPAAC) allows preparation of conjugates under copper-free conditions that are suitable for use in live cells and animals. ${ }^{16-18}$ Azidefunctionalised amino acids can be incorporated into proteins using the methionine surrogate azidohomoalanine (Aha). ${ }^{19,20}$ In methionine-depleted cultures, Aha is activated by methionyltRNA synthetase of methionine auxotroph Escherichia coli and replaces methionine in translated proteins. In this communication we report a novel azido-CTB toxoid and demonstrate its utility for neuronal tracing in vivo. Azido-CTB can be made in any lab with protein production facilities and a variety of molecules may be readily conjugated in a site-specific manner to suit individual needs.

The isosteric replacement of methionine with Aha at the surface of CTB required the creation of a new mutant toxoid. Three sequential rounds of site-directed mutagenesis were used to replace all the native methionine residues with leucines 

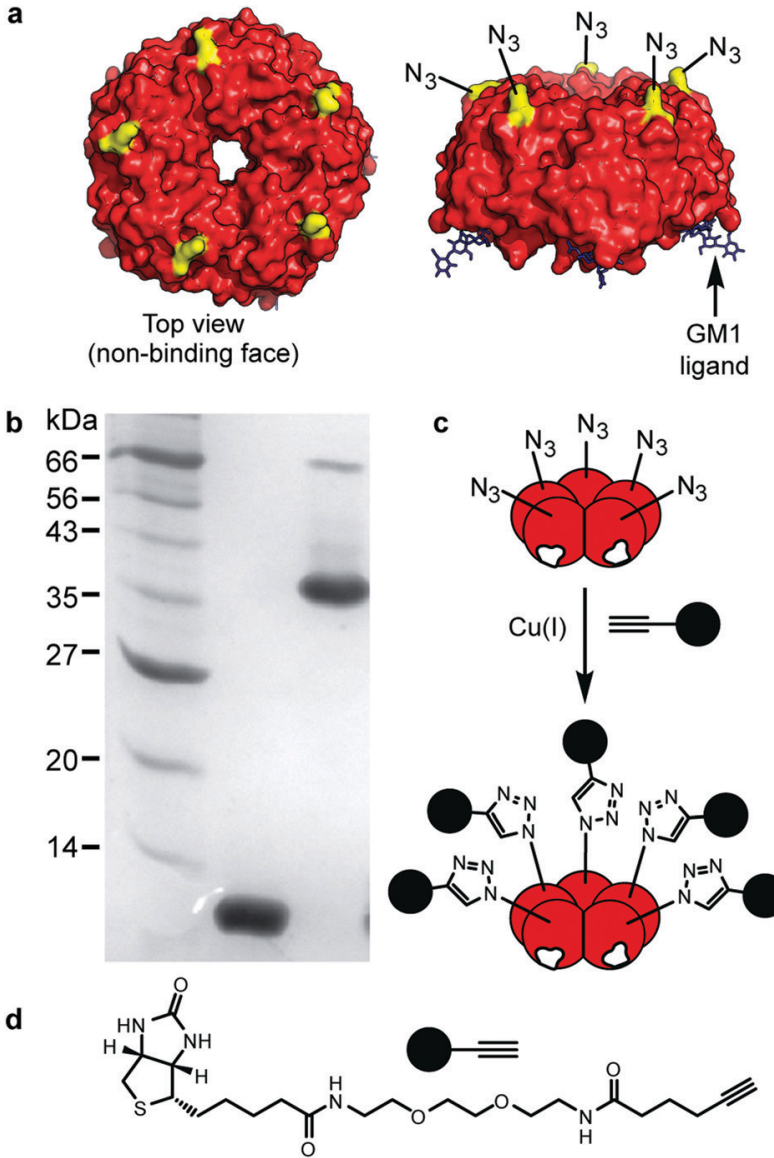

Fig. 1 Design and structure of azido-CTB. (a) Crystal structure of CTB (red) bound to GM1 (black). Residue K43 (yellow). (b) SDS-PAGE gel (12\% acrylamide) of azido-CTB elution fraction after Ni-NTA column (left to right: molecular weight markers; boiled azido-CTB; non-boiled sample showing pentameric azido-CTB. (c) Cartoon showing cycloaddition of azido-CTB with (d) a biotin-functionalised alkyne.

(M37L, M68L, M101L) and a surface exposed lysine residue with methionine (K43M) (Fig. S1, ESI $\dagger$ ). As the K43M mutation is on the non-binding face of the protein (Fig. 1a), future chemical modifications at this site were predicted not to interfere with CTB-GM1 binding. Methionine auxotroph E. coli B834 Gold (DE3) cells were transformed with the new construct, pSAB2.3 (Azido), and azido-CTB was overexpressed using a defined growth medium supplemented with Aha. ${ }^{19-21}$ The azido-CTB was purified using Ni-affinity chromatography and size exclusion chromatography before analysis by SDS-PAGE and mass spectrometry (Fig. S2, ESI $\dagger$ ). For the wild type CTB protein, the tetra-mutant CTB is sufficiently stable to migrate as a pentamer during SDS-PAGE unless the sample has been boiled prior to loading (Fig. 1b). While the native CTB normally gives rise to a protein band around $45 \mathrm{kDa}$ on a gel, azido-CTB is more negatively charged than native CTB which likely explains why it ran faster (apparent mass $<40 \mathrm{kDa}$ ).

The ability of the purified azido-CTB protein to enter motor neurons, transport in a retrograde manner to the brainstem and to be detected in vivo via CuAAC, was tested using tongue injections in mice. Azido-CTB successfully traced the motor
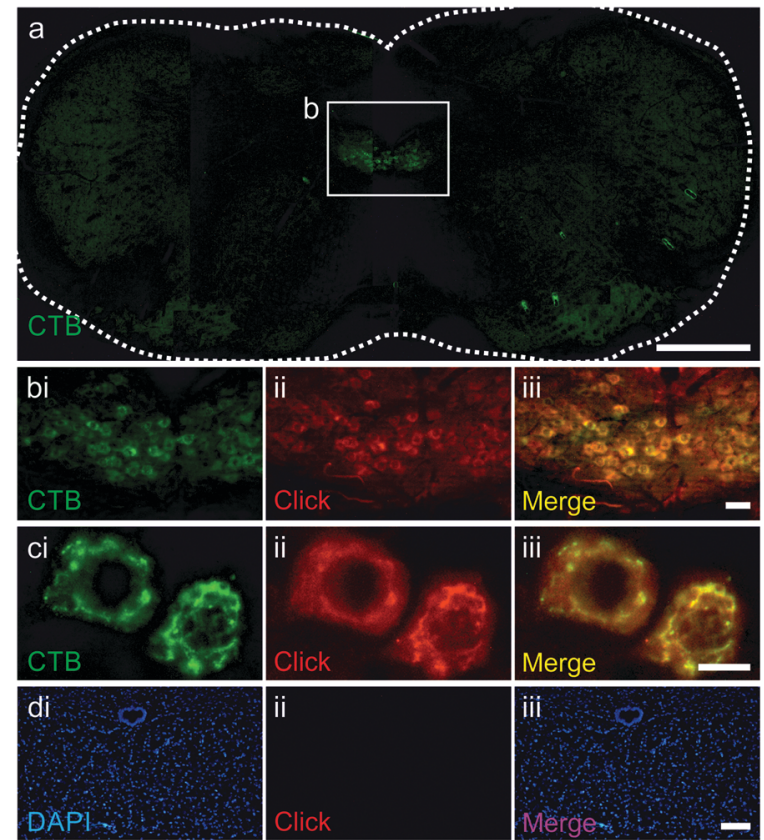

Fig. 2 Azido-CTB as a neuronal tracer detected by CuAAC. (a) Whole brainstem section stained with anti-CTB antibody (green), hypoglossal nucleus shown in white box. ( $b$ and $c$ ) Hypoglossal nucleus stained with anti-CTB antibody (green, b-ci), CuAAC (red, b-cii) and merged (b-ciii). (d) Control brainstem slice from non-injected mouse stained with the nuclear marker DAPI (blue, di), CuAAC (red, dii) and merged (diii). Scale bars: $\mathrm{a}=500 \mu \mathrm{m} ; \mathrm{b}=50 \mu \mathrm{m} ; \mathrm{c}=10 \mu \mathrm{m} ; \mathrm{d}=100 \mu \mathrm{m}$.

neurons of the hypoglossal nucleus that innervate the tongue; it was detected using CuAAC with a biotinylated alkyne (Kerafast) followed by Alexa-555 labelled streptavidin (streptavidin-555) in paraformaldehyde-fixed brain tissue (Fig. 2b-cii). Streptavidin labelling (shown in red) overlapped with immunohistochemical detection for CTB using an anti-CTB primary antibody and secondary antibody labelled with Alexa-488), it was detected in the same cells and to the same extent (Fig. 2a-ci). Control brainstem slices with no azido-CTB injection undergoing the click reaction and detection with streptavidin-555 gave no positive cells, verifying that the signal in injected tissue is from azido-CTB (Fig. 2d). Azido-CTB can therefore be used as a neuronal tracer. If used in conjunction with immunohistochemistry to identify other proteins such as ion channels and receptor subunits within the tissue, it will reduce limitations to the primary antibody species that can be used since CTB is detected without antibodies. Furthermore, azido-CTB has the potential to be used to make a fluorescently tagged tracer for delivery with any fluorescent molecule containing an alkyne group.

To further extend the flexibility of azido-CTB, we used its azide moiety for conjugation with nanoparticles prior to neuronal tracing. Correlative light and electron microscopy provides a powerful and complementary alternative to immunohistochemistry for studying sub-cellular localisation of probes. In this context, targeted nanoparticles can be used as nucleation sites for deposition of silver to facilitate their detection without the need to permeabilise the tissue. We therefore investigated the conjugation of $\mathrm{CdSe} / \mathrm{ZnS}$ core/shell nanoparticles to azido-CTB 


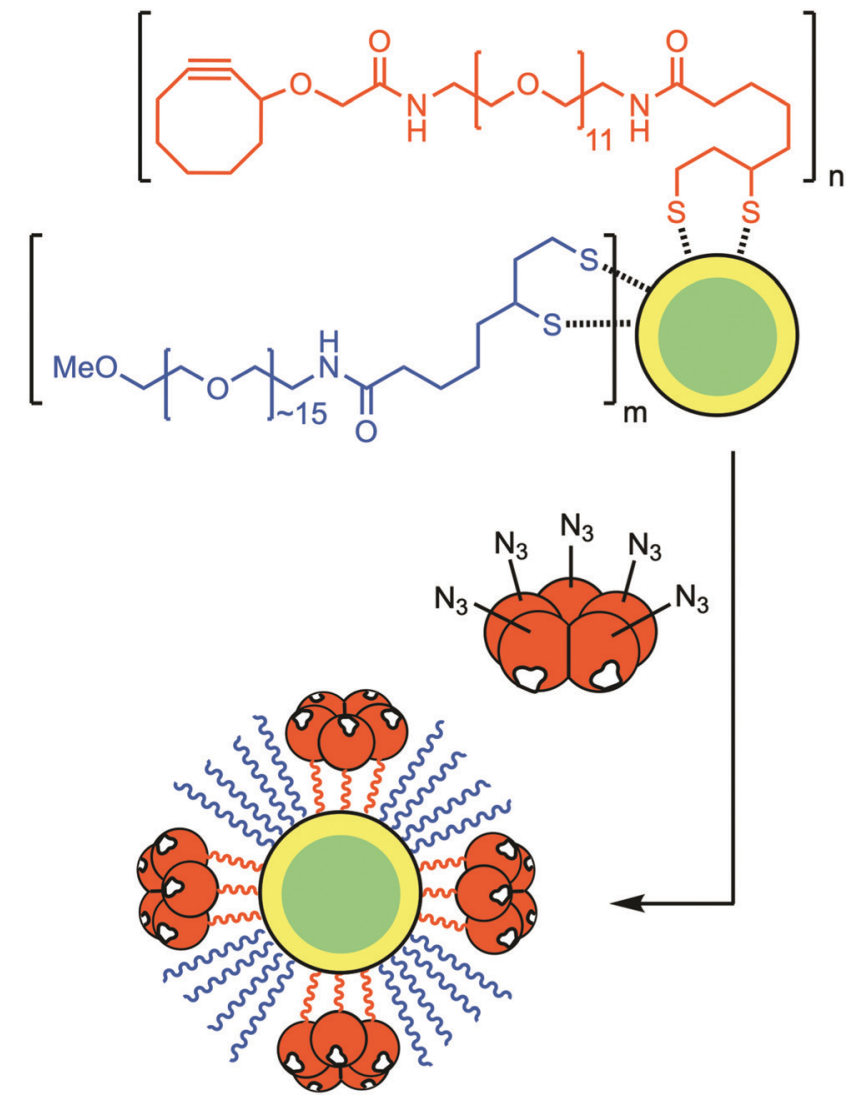

Fig. 3 Preparation of CTB-nanoparticle-based neuronal tracers for detection by electron microscopy. Azido-CTB was conjugated via SPAAC to dihydrolipoic acid-PEG ${ }_{11}$-cyclooctyne-functionalised CdSe/ZnS nanoparticles prepared with $10 \%(n: m=1: 9)$ or $25 \%(n: m=1: 3)$ of the active ligand. It is assumed that the final ligand composition on the nanoparticles is comparable to that of the mixture of DHLA ligands used in their preparation.

and their ability to target neurons in vivo. The nanoparticles were first functionalised with a ligand mixture of dihydrolipoic acid (DHLA)-PEG ${ }_{11}$-cyclooctyne and a DHLA-PEG $\sim 15$-OMe spacer via cap-exchange at a total ligand:nanoparticle molar ratio of $3000: 1$, with $10 \%$ or $25 \%$ of the ligands being DHLA-PEG ${ }_{11^{-}}$ cyclooctyne ligand (Fig. 3). ${ }^{2-24}$ Azido-CTB was then directly conjugated to the nanoparticles by SPAAC as confirmed by gel electrophoresis (ESI, $\dagger$ Fig. S3). Site-specific biorthogonal labelling of CTB with the nanoparticles means that the binding face of CTB remains unobstructed and thus endocytosis of the complex should be unaltered, unlike other studies using random derivations. ${ }^{25}$

The CTB-functionalised nanoparticles $(2 \mu \mathrm{L}$ of $2.4 \mu \mathrm{M})$ were injected via the tongue in mice to assess whether the nanoconjugates could be endocytosed and transported in a retrograde direction in vivo. Silver enhanced brainstem slices revealed that the nano-conjugates were in neurons of the hypoglossal nucleus after injection (Fig. 4a). Staining was found optimal when using azido-CTB conjugated to nanoparticles prepared using $25 \%$ of the active DHLA-PEG $11^{-}$-cyclooctyne ligand as shown in Fig. 3. Given the reduced amount of

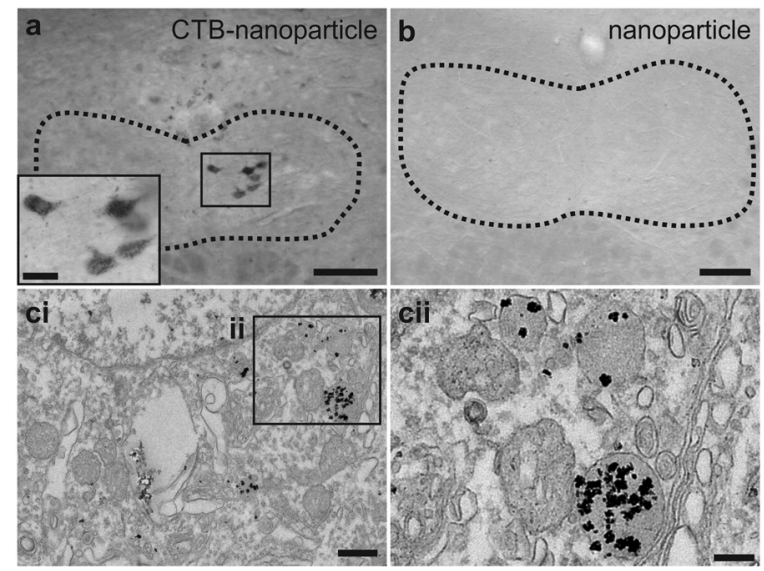

Fig. 4 Use of CTB-nanoparticles as neuronal tracers for detection by electron microscopy. (a) Hypoglossal nucleus (dotted lines) having undergone silver enhancement, inset shows enlarged positive neurons. (b) Control brainstem slice from uninjected mouse having undergone silver enhancement. (c) Electron microscopy of hypoglossal nucleus cell (ci) and lysosomes inside that cell (cii). Labelling is confined to these structures. Scale bars: $\mathrm{a}$ and $\mathrm{b}=100 \mu \mathrm{m}$; $\mathrm{a}$ inset $=25 \mu \mathrm{m} ; \mathrm{ci}=0.5 \mu \mathrm{m}$; cii $=0.2 \mu \mathrm{m}$.

CTB-nanoparticles delivered versus azido-CTB, there are fewer labelled cells. These nanoparticles were not detected in mice injected with just nanoparticles (Fig. 4b), indicating that they cannot transport in a retrograde manner without CTB. The silver enhanced slices were then prepared for EM where puncta corresponding to the metal deposits were found to be mainly localised to lysosomes (Fig. 4c). While CTB is known to travel in a retrograde manner to the Golgi, ${ }^{26,27}$ the appearance of CTB-nanoparticle conjugates in the lysosome is also in agreement with other studies, ${ }^{28,29}$ and may be the final fate of CTB after longer periods in vivo. Nonetheless, the easy application of azido-CTB as a nanoparticle-conjugate could further improve its use as a neuronal tracer for EM analysis. It does not require cellular permeabilisation, and thus avoids damage to cellular membranes, which occurs when using agents such as Triton $\mathrm{X}-100$ or ethanol to allow penetrance of antibodies. The easy detection of CTB-nanoparticle conjugates via correlated light microscopy and EM, thus provides a much wider scope for possible experiments with tissue. These experiments highlight that alkynated substances can be readily and efficiently "clicked" onto azido-CTB prior to injection, increasing the possibility for rapid testing of CTB as a powerful delivery vehicle to carry different cargoes into neurons.

In summary, azido-CTB presents a useful tool for diversifying and enhancing its use as both a tracer and a delivery vehicle compared to current commercially available CTB conjugates due to ease of directed conjugation via CuAAC and SPAAC. Azido-CTB may be further developed to create interchangeable conjugated proteins via click chemistry; it could deliver multiple cargoes at once, or cargo along with a reporter dye or even a nanoparticle. This has been proven with CTB-nanoparticle conjugates produced from azido-CTB; where the nanoparticles were detectable after injection in vivo. Azido-CTB presents a diverse tool for tracing that is subsequently detected both with fluorescence 
and EM, for use in live and fixed tissue, and for use in extended time-lapse experiments. Moreover, other theranostic functions can be readily incorporated via conjugating to other functional nanoparticles (e.g. magnetic nanoparticles for magnetic resonance imaging; ${ }^{30}$ gold nanorods for photothermal treatment and/or photoacoustic imaging). ${ }^{31,32}$ With the increasing array of molecules and nanoparticles able to be 'clicked' to azides, there is a wide scope of opportunity for azido-CTB to be utilised for efficient and rapid attachment of novel tracers, cargoes and/or incorporating with multiple theranostic functions.

The authors thank the BBSRC for funding through Doctoral Training Partnership BB/J014443/1, and research grant BB/ M005666/1, and EPSRC for funding through Doctoral Training Partnership EP/M50807X/1, with equipment support from the Wellcome Trust grants WT094232MA and 094232/Z/10/Z.

\section{Conflicts of interest}

There are no conflicts to declare.

\section{References}

1 T. Beddoe, A. W. Paton, J. Le Nours, J. Rossjohn and J. C. Paton, Trends Biochem. Sci., 2010, 35, 411-418.

2 E. A. Merritt, S. Sarfaty, F. van den Akker, C. L'Hoir, J. A. Martial and W. G. Hol, Protein Sci., 1994, 3, 166-175.

3 B. Lindh, H. Aldskogius and T. Hökfelt, Histochemistry, 1989, 92, 367-376.

4 P. J. Dederen, A. A. Gribnau and M. H. Curfs, Histochem. J., 1994, 26, 856-862.

5 A. Gramsbergen, J. Ijkema-Paassen and M. F. Meek, Exp. Neurol., 2000, 161, 183-193.

6 P.-H. Luppi, K. Sakai, D. Salvert, P. Fort and M. Jouvet, Brain Res., 1987, 402, 339-345.

7 R. Flink and J. Westman, J. Comp. Neurol., 1986, 250, 265-281.

8 F. Datiche, P. H. Luppi and M. Cattarelli, Brain Res., 1995, 671, 27-37.

9 N. Luquin, S. Sierra, A. J. Rico, V. Gomez-Bautista, E. Roda, L. ContePerales, R. Franco, P. McCormick, J. L. Labandeira-Garcia and J. L. Lanciego, Neurobiol. Dis., 2012, 47, 347-357.

10 K. E. Fasanella, J. A. Christianson, R. S. Chanthaphavong and B. M. Davis, J. Comp. Neurol., 2008, 509, 42-52.
11 W. L. Conte, H. Kamishina and R. L. Reep, Nat. Protoc., 2009, 4, 1157-1166.

12 H. Liu, I. J. Llewellyn-Smith and A. I. Basbaum, J. Histochem. Cytochem., 1995, 43, 489-495.

13 V. V. Rostovtsev, L. G. Green, V. V. Fokin and K. B. Sharpless, Angew. Chem., Int. Ed., 2002, 41, 2596-2599.

14 C. W. Tornoe, C. Christensen and M. Meldal, J. Org. Chem., 2002, 67, 3057-3064.

15 L. Li and Z. Zhang, Molecules, 2016, 21, 1393.

16 J. Dommerholt, F. P. J. T. Rutjes and F. L. van Delft, Top. Curr. Chem., 2016, 374, 16.

17 E. M. Sletten and C. R. Bertozzi, Acc. Chem. Res., 2011, 44, 666-676.

18 J. C. Jewett and C. R. Bertozzi, Chem. Soc. Rev., 2010, 39, 1272-1279.

19 K. L. Kiick, E. Saxon, D. A. Tirrell and C. R. Bertozzi, Proc. Natl. Acad. Sci. U. S. A., 2002, 99, 19-24.

20 F. Tobola, E. Sylvander, C. Gafko and B. Wiltschi, Interface Focus, 2019, 9, 20180072.

21 B. Wiltschi, in Synthetic Gene Networks: Methods and Protocols, ed. W. Weber and M. Fussenegger, Humana Press, Totowa, NJ, 2012, pp. 211-225.

22 Y. Guo, C. Sakonsinsiri, I. Nehlmeier, M. A. Fascione, H. Zhang, W. Wang, S. Pöhlmann, W. B. Turnbull and D. Zhou, Angew. Chem., Int. Ed., 2016, 55, 4738-4742.

23 Y. Guo, I. Nehlmeier, E. Poole, C. Sakonsinsiri, N. Hondow, A. Brown, Q. Li, S. Li, J. Whitworth, Z. Li, A. Yu, R. Brydson, W. B. Turnbull, S. Pöhlmann and D. Zhou, J. Am. Chem. Soc., 2017, 139, 11833-11844.

24 B. C. Mei, K. Susumu, I. L. Medintz, J. B. Delehanty, T. J. Mountziaris and H. Mattoussi, J. Mater. Chem., 2008, 18, 4949-4958.

25 S. K. Chakraborty, J. A. J. Fitzpatrick, J. A. Phillippi, S. Andreko, A. S. Waggoner, M. P. Bruchez and B. Ballou, Nano Lett., 2007, 7, 2618-2626.

26 D. C. Machin, D. J. Williamson, P. Fisher, V. J. Miller, G. C. Wildsmith, J. F. Ross, C. Wasson, A. MacDonald, B. I. Andrews, D. Ungar, W. B. Turnbull and M. E. Webb, ChemRxiv, 2019, DOI: 10.26434/chemrxiv.9125201.v1.

27 W. I. Lencer, T. R. Hirst and R. K. Holmes, Biochim. Biophys. Acta, 1999, 1450, 177-190.

28 W. A. Walker, M. Tarannum and J. L. Vivero-Escoto, J. Mater. Chem. $B, 2016,4,1254-1262$.

29 K. C. Joseph, S. U. Kim, A. Stieber and N. K. Gonatas, Proc. Natl. Acad. Sci. U. S. A., 1978, 75, 2815.

30 T.-H. Shin, Y. Choi, S. Kim and J. Cheon, Chem. Soc. Rev., 2015, 44, 4501-4516.

31 Y.-S. Chen, Y. Zhao, S. J. Yoon, S. S. Gambhir and S. Emelianov, Nat. Nanotechnol., 2019, 14, 465-472.

32 W. Zhang, F. Wang, Y. Wang, J. Wang, Y. Yu, S. Guo, R. Chen and D. Zhou, J. Controlled Release, 2016, 232, 9-19. 